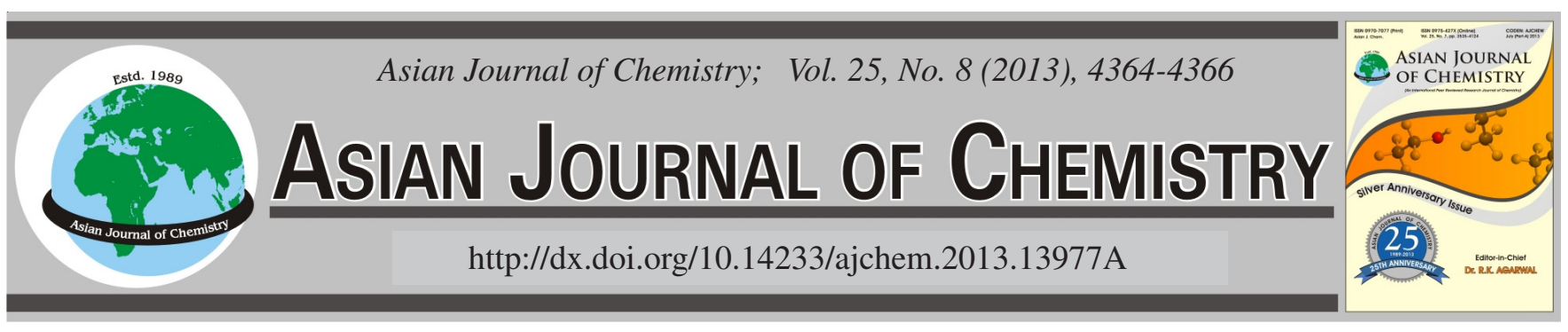

\title{
Variation of Carotenoid Content in Agastache rugosa and Agastache foeniculum
}

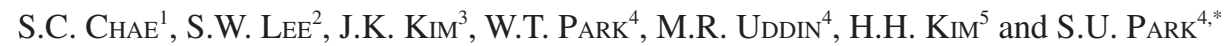

${ }^{1}$ Department of Horticultural Science, College of Industrial Sciences, Kongju National University, 1 Daehoe-ri, Yesan-kun, Chungnam, 340-720, South Korea

${ }^{2}$ Herbal Crop Research Team, International Institute of Horticultural \& Herbal Science, RDA, Eumseong-gun, Chungcheongbuk-do 369-873, South Korea

${ }^{3}$ National Academy of Agricultural Science, Rural Development Administration, Suwon 441-707, South Korea

${ }^{4}$ Department of Crop Science, Chungnam National University, 79 Daehangno, Yuseong-Gu, Daejeon, 305-764, South Korea

${ }^{5}$ Department of Well-being Resources, Sunchon National University, 413 Jungangno, Suncheon, Jeollanam-do, 540-742, South Korea

*Corresponding author: Fax: +82 42 8222631; Tel: +82 42 8215730; E-mail: supark@cnu.ac.kr

(Received: 27 April 2012;

Accepted: 11 February 2013)

AJC-12965

The present study describes the variation in carotenoid content between different organs (leaf, flower, stem and root) of Agastache rugosa
and Agastache foeniculum species. Analysis of distinct parts of the two Agastache spp revealed the presence of 6 carotenoids-violaxanthin,
antheraxanthin, lutein, zeaxanthin, $\alpha$-carotene and $\beta$-carotene. However, the levels of these carotenoids varied significantly among the
different plant organs. All 6 carotenoids were observed in the leaves of both species. Moreover, the amount of carotenoids in the leaves
was much higher than in the stem, flower or root. The carotenoid content was higher in all parts of $A$. rugosa compared with A. foeniculum.
In particular, the levels of $\alpha$-carotene was 9.6 -fold higher in the leaves of A. rugosa than that in the leaves of A. foeniculum. The levels of
lutein and $\beta$-carotene in the stem of A. rugosa were 2.8 and 2.1 -fold higher, respectively, compared with those in the stem of A.foeniculum.
Thus, the amount of carotenoids varied widely between the different parts of Agastache spp. The variation in carotenoid content between
the two species was significant.

Key Words: Agastache spp, Carotenoids, Leaf, Stem, Flower, Root.

ᄂ ----------------------------------$\lrcorner$

\section{INTRODUCTION}

Carotenoids are the second most abundant pigment and a diverse group of more than 750 naturally occurring red, orange and yellow pigments ${ }^{1}$ that accumulate in the plastids of leaves, flowers and fruits, thereby attracting pollinators and seed dispersal agents ${ }^{2}$. In plant, carotenoids play vital roles in photosynthesis, photomorphogenesis and photoprotection ${ }^{3,4}$. Carotenoids are a group of phytonutrients that can lower the bad cholesterol and triglyceride levels in the blood stream. Moreover, carotenoids are the precursors of an important plant hormone, abscisic $\operatorname{acid}^{5}$, which is involved in regulation of the plant stress response.

A number of carotenoids serve as precursors of vitamin A and act as antioxidants that could protect the human body from the harmful functions of the free radicals may prevent degenerative diseases ${ }^{6}$. A diet with carotenoid-rich vegetables and fruit has been shown to protect against some cancers, heart diseases, cataracts and UV-induced skin damage ${ }^{7}$.

Agastache rugosa Kuntze is a perennial herb of the mint family (Labiatae). It is widely distributed in East- and Southeast Asian countries. Since a long time this herb is traditionally used in Chinese medicine for the treatment of cholera, vomiting and miasma and has been reported to possess antitumor, HIV integrase inhibitory, antifungal and cytotoxic activities. It is mentionable that the leaves of A. rugosa are used in cooking to season fish-based foods and its flowers are a prime source of honey ${ }^{8-10}$.

Another species of Agastache foeniculum (Pursh) Kuntze is also a perennial herb belongs to the same family. The origin of this herb is around north-central and northern regions of North America and was traditionally used by Native Americans as a medicine to treat cough, fevers, wounds and diarrhea. The special feature of this herb has anise-scented leaves that are used as a seasoning, to prepare tea and in potpourri. Moreover, the characteristics purple flower spike attracts bees, which make a fragrant honey from the nectar ${ }^{11,12}$. Therefore, in the present paper, we analyzed the carotenoid content in different organs (i.e., the leaf, stem, flower and root) of A. rugosa and A. foeniculum.

\section{EXPERIMENTAL}

Plant materials: A. rugosa and A. foeniculum were grown in a greenhouse at an experimental farm of Kongju National 
University (Yesan, Korea). Plant materials were excised from mature plants and dissected into flowers, leaves, stems and roots. The samples were immediately frozen in liquid nitrogen and stored at $-80{ }^{\circ} \mathrm{C}$ until further use for carotenoid analysis.

Extraction and HPLC analysis of carotenoids: Carotenoids were extracted from Agastache spp. samples (0.1 g) with $3 \mathrm{~mL}$ of ethanol containing $0.1 \%$ ascorbic acid (w/v). This mixture was vortexed for $20 \mathrm{~s}$ and incubated in a water bath at $85{ }^{\circ} \mathrm{C}$ for $5 \mathrm{~min}$. Subsequently, $120 \mu \mathrm{L}$ of potassium hydroxide $(80 \% \mathrm{w} / \mathrm{v})$ was added to saponify any potentially interfering oils. After vortexing and incubating at $85^{\circ} \mathrm{C}$ for 10 min, the samples were placed on ice and $1.5 \mathrm{~mL}$ of cold deionized water and $0.05 \mathrm{~mL}$ of $\beta$-apo-8-carotenal $(12.5 \mu \mathrm{g}$ $\mathrm{mL}^{-1}$; an internal standard) were added. The carotenoids were subsequently extracted twice with $1.5 \mathrm{~mL}$ of hexane and centrifuged at $1200 \mathrm{~g}$ following each extraction in order to separate the layers. Finally, the extracts were freeze dried under a stream of nitrogen gas and resuspended in 50:50 (v/v) dichloromethane/methanol. For HPLC analysis, the carotenoids were separated on an agilent 1100 HPLC system using a $\mathrm{C}_{30}$ YMC column $(250 \mathrm{~mm} \times 4.6 \mathrm{~mm}, 3 \mu \mathrm{m}$; Waters Corporation, Milford, MA) and detected using a photodiode array (PDA) detector at $450 \mathrm{~nm}$. Solvent A consisted of methanol/water $(92: 8 \mathrm{v} / \mathrm{v})$ with $10 \mathrm{~mm}$ ammonium acetate, whereas solvent B comprised $100 \%$ methyl tert-butyl ether (MTBE). The flow rate was maintained at $1 \mathrm{~mL} \mathrm{m^{-1 }}$ and samples were eluted with the following gradient: $0 \mathrm{~min}, 83 \% \mathrm{~A} / 17 \% \mathrm{~B} ; 23 \mathrm{~min}$, $70 \% \mathrm{~A} / 30 \% \mathrm{~B} ; 29 \mathrm{~min}, 59 \% \mathrm{~A} / 41 \% \mathrm{~B} ; 35 \mathrm{~min}, 30 \% \mathrm{~A} / 70$ $\% \mathrm{~B} ; 40 \mathrm{~min}, 30 \% \mathrm{~A} / 70 \% \mathrm{~B} ; 44 \mathrm{~min}, 83 \% \mathrm{~A} / 17 \% \mathrm{~B}$; and 55 $\min , 83 \% \mathrm{~A} / 17 \% \mathrm{~B}$.

\section{RESULTS AND DISCUSSION}

The variations in carotenoid content between different parts of Agastache spp. (A. rugosa and A. foeniculum) are shown in Table-1. Analysis of the different parts of Agastache spp., revealed the presence of 6 different carotenoids i.e., violaxanthin, antheraxanthin, lutein, zeaxanthin, $\alpha$-carotene and $\beta$-carotene. However, the levels of these carotenoids varied significantly between the different organs of Agastache spp. In addition, while all 6 carotenoids were found in the flowers of $A$. rugosa, violaxanthin was absent from the flowers of $A$. foeniculum. Moreover, the levels of $\beta$-carotene, antheraxanthin, zeaxanthin and lutein were 1.97, 1.7, 1.6 and 1.5-fold higher, respectively, in the flowers of $A$. rugosa than in those of $A$. foeniculum. The carotenoid content in the leaves of both species of Agastache was much higher than in any other part of the plant. Furthermore, while all 6 carotenoids were observed in the leaves of both species of Agastache, the content of all carotenoids was greater in the leaves of A. rugosa. In particular, the amount of $\alpha$-carotene in A. rugosa leaves was 9.6-fold higher than that in A. foeniculum leaves. The levels of zeaxanthin, violaxanthin, $\beta$-carotene, lutein and antheraxanthin were $2.3,2.1,1.9,1.5$ and 1.4-fold higher, respectively, in the leaves of A. rugosa than those in A. foeniculum leaves. Among the 6 carotenoids, 3 carotenoids i.e., lutein, zeaxanthin and $\beta$-carotene were found in the stem of A. rugosa, whereas only 2 carotenoids i.e., lutein and $\beta$-carotene were observed in the stem of A. foeniculum. Moreover, the overall level of carotenoids was higher in the stem of A. rugosa. Specially, the levels of lutein and $\beta$-carotene were 2.8 and 2.1-fold higher, respectively, in the stem of $A$. rugosa than those in the stem of A. foeniculum. Furthermore, the carotenoid content was higher in the stem than in the leaf for both species of Agastache. The amount of carotenoids in the roots of both Agastache spp. was much lower than in other parts of the plants. Among the 6 carotenoids identified, only 2 carotenoids i.e., lutein and $\beta$-carotene were found in the roots of both species of Agastache. Similar to the carotenoid content in the stem, the overall level of carotenoids was higher in the roots of A. rugosa. In particular, the amount of lutein was 1.7-fold higher in the roots of A. rugosa compared with those of A. foeniculum. It was previously reported that there was significant variation in oil content of the different lines of Agastache spp., ranging from 0.07 to 2.73 (per cent volume/dry weight) for leaves and from 0.10 to 3.00 (per cent volume/dry weight) for flowers ${ }^{13}$. Variation in the essential oil composition was high among lines of $A$. foeniculum but low among lines of A. rugosa ${ }^{13}$. Calendula officinalis L. is a medicinal plant that accumulates large amounts of carotenoids in its inflorescences. The yellow-toorange colour of inflorescences is mostly due to carotenoids and the shade is dependent on pigments content and profile ${ }^{14-16}$. Some works refer to qualitative aspects (separation and identification of carotenoids) and others to quantitative determina$\operatorname{tion}^{17,18}$ (total carotenoid content). It was found the variation of carotenoid contents in four varieties of Calendula officinalis L. flowers ${ }^{19}$. The carotenoids composition of petals, pollens, leaves and steams of calendula was investigated by HPLC and found difference among the organs ${ }^{20}$. In present study we also found a difference among the organs that agree with the resulrs of Bako et al. ${ }^{20}$.

The carotenoid content in the different organs of Agastache spp. varied widely. However, the leaves were found to possess the highest levels of all 6 carotenoids analyzed. In addition, the species also influenced the carotenoid content

TABLE- 1

CAROTENOID CONTENT IN Agastache rugosa AND Agastache foeniculum

\begin{tabular}{cccccccc}
\hline \multirow{2}{*}{ Agastache spp. } & \multirow{2}{*}{ Organs } & \multicolumn{5}{c}{ Carotenoids $\left(\mu \mathrm{g} \mathrm{g}^{-1}\right)$} \\
\cline { 3 - 8 } & & Violaxanthin & Antheraxanthin & Lutein & Zeaxanthin & $\alpha$-Carotene & $\beta$-Carotene \\
\hline \multirow{3}{*}{$\begin{array}{c}\text { Agastache } \\
\text { rugosa }\end{array}$} & Flower & $0.9 \pm 0.0$ & $0.5 \pm 0.0$ & $33.3 \pm 2.4$ & $3.1 \pm 0.2$ & $0.4 \pm 0.0$ & $36.1 \pm 1.4$ \\
& Leaf & $11.7 \pm 3.4$ & $5.5 \pm 0.7$ & $277.1 \pm 61.2$ & $29.4 \pm 4.1$ & $4.8 \pm 0.1$ & $499.2 \pm 22.2$ \\
& Stem & 0 & 0 & $94.3 \pm 11.7$ & $8.0 \pm 0.0$ & 0 & $65.0 \pm 7.3$ \\
\hline \multirow{3}{*}{ Agastache } & Root & 0 & 0 & $2.7 \pm 0.6$ & 0 & 0 & $1.1 \pm 0.5$ \\
foeniculum & Flower & 0 & $0.3 \pm 0.0$ & $22.1 \pm 2.42$ & $1.9 \pm 0.3$ & $0.4 \pm 0.0$ & $18.3 \pm 1.4$ \\
& Leaf & $5.6 \pm 3.4$ & $3.8 \pm 0.7$ & $189.7 \pm 61.2$ & $13.04 \pm 4.1$ & $0.5 \pm 0.1$ & $260.9 \pm 22.2$ \\
& Stem & 0 & 0 & $33.7 \pm 11.7$ & 0 & 0 & $31.1 \pm 7.3$ \\
\hline & Root & 0 & 0 & $1.6 \pm 0.6$ & 0 & 0 & $1.1 \pm 0.6$ \\
\hline
\end{tabular}


with $A$. rugosa containing higher amounts of carotenoids in all its organs. Therefore, the leaves of A. rugosa may have commercial or medicinal applications, particularly in the production of carotenoids.

\section{ACKNOWLEDGEMENTS}

This research was supported by Basic Science Research Program through the National Research Foundation of Korea (NRF) funded by the Ministry of Education, Science and Technology (20110010231).

\section{REFERENCES}

1. G. Britton, S. Liaaen-Jensen and H. Pfander, Carotenoids Handbook, Berlin, Germany (2004).

2. C.A. Howitt and B.J. Pogson, Plant Cell Environ., 29, 435 (2006)

3. G.E. Bartley and P.A. Scolnik, Plant Cell, 7, 1027 (1995).

4. J. Penuelas and S. Munne-Bosch, Trends Plant Sci., 10, 166 (2005).

5. J. Hirschberg, Curr. Opin. Plant Biol., 4, 210 (2001).

6. E. Giovannucci, J. Nat. Cancer Inst., 91, 317 (1999).

7. P. Botella-Pavía and M. Rodríguez-Concepción, Physiol. Plant., 126, 369 (2006).
8. J.J. Hong, J.H. Choi, S.R. Oh, H.K. Lee, J.H. Park, K.Y. Lee, J.J. Kim, T.S. Jeong and G.T. Oh, FEBS Lett., 495, 142 (2001).

9. S. Shin and C.A. Kang, Lett. Appl. Microbiol., 36, 111 (2003).

10. H.M. Oh, Y.J. Kang, S.H. Kim, Y.S. Lee, M.K. Park, J.M. Heo, J. Sun, H.J. Kim, E.S. Kang, H.J. Kim, H.G. Seo, J.H. Lee, H.S. Yun-Choi and K.C. Chang, Arch. Pharm. Res., 28, 305 (2005).

11. I. Nykänen, Y. Holm and R. Hiltunen, Planta Med., 55, 314 (1989).

12. R.G. Fuentes Granados, M.P. Widrlechner and L.A. Wilson, J. Herbs Spices Med. Plants, 6, 69 (1998).

13. D.J. Charles, J.E. Simon and M.P. Widrlechnert, J. Agric. Food Chem., 39, 1946 (1991).

14. G. Toth and J. Szabolcs, Phytochemistry, 20, 2411 (1981).

15. B.V. Milborrow, I.E. Swift and A.O. Nelting, Phytochemistry, 21, 2853 (1982).

16. G. Petri and G. Lemberkovics, Acta Pharm. Hung., 64, 87 (1994).

17. U. Bomme, J. Holzl and E. Schneider, Herba. Hung., 29, 19 (1997).

18. R. Piccaglia, M. Marotti, G. Chiavari and N. Gandini, Flav. Fragr. J., 12, 85 (1997).

19. A. Pintea, C. Bele, S. Andrei and C. Socaciu, Acta Biol. Szeged., 47, 37 (2003).

20. E. Bako, J. Deli and G. Toth, J. Biochem. Biophys. Methods, 53, 241 (2002). 